\title{
On the Effects of Balun on Small Antennas Performance for Animal Health- Monitoring and Tracking
}

\author{
A. Alemaryeen ${ }^{1}$ and S. Noghanian² \\ ${ }^{1}$ Department of Communication, Electronics and Computer Engineering, Tafila Technical University, Tafila 66110, Jordan \\ ${ }^{2}$ Wafer LLC., Danvers, MA 01923 USA, and American Public University Systems, Charles Town, VW \\ Corresponding author: A. Alemaryeen (e-mail: ala.alemaryeen@ttu.edu.jo).
}

\begin{abstract}
This paper presents the performance evaluation of a sleeve Balun integration in the design of a flexible loop antenna for wildlife health monitoring and tracking applications. To verify the design concept, an experimental antenna is designed, fabricated, and measured in free-space and muscle mimicking phantom. Moreover, investigations are carried out for wearable and implanted antennas in planar and conformal arrangements. In free-space, the antenna is operating within the industrial, scientific, and medical ISM $5.8 \mathrm{GHz}$ band. Balun integration in the antenna design efficiently chokes the currents excited on the outer surface of the feeding cable and provides a good impedance match between antenna and feed line, as demonstrated by simulation and measurement results. On the other hand, in phantom, the antenna has a wide bandwidth characteristic that covers the most used frequency bands for in-body devices. Balun integration, in this case, showed a negligible effect on antenna's matching properties for two studied implantation depths; $2.5 \mathrm{~cm}$ and $5 \mathrm{~cm}$. The proposed study offers a promising guideline in the design and realization of wearable and implanted antennas.
\end{abstract}

INDEX TERMS Balun, coaxial fed antennas, conformal antennas, implantable antennas, transmission line.

\section{INTRODUCTION}

D EVELOPMENT of modern wireless communication systems is of great importance, especially with the witnessed technological revolution. Wearable and implantable technologies, satisfy different demands for continuous, convenient, and flexible access to up-to-date information [1]. These technologies have successfully been used for animals' health monitoring and tracking of different species such as measuring canine vital signs [2], detecting the presence of viruses and pathogens in the poultry industry [3], tracking birds [4] and bats [5], and pregnancy detection in cows by monitoring temperature changes in the body [6]. Advantages of using these technologies for animals can be seen through providing a timely diagnosis of diseases in animals, eventually decreasing economic losses, prevention of road-side accidents, understanding complex biological processes such as social interactions and migration, and prevention illegal hunting of endangered species [7]-[8]. Efficiency of such technologies depends on the radio frequency front ends which are highly required with enhanced performance in convenience, low cost, and compact size [9]. Recently, numerous research work has been conducted for improving the performance of the antenna, that can efficiently radiate and accept electromagnetic $(\mathrm{EM})$ waves and utilizing this antenna in the system.

Most antennas used for wearable and implantable applications are either fed by or constructed out of coaxial cables. Due to their unbalanced nature, coaxial cables allow returning currents to flow on the outer surfaces of the outer conductors [10]. If these currents are not properly suppressed, the radiation associated with them will degrade the impedance matching between the antenna and the source and causes unwanted heating of healthy tissue, in case of implanted antennas, along the cable shafts. Consequently, coax-fed antennas are commonly equipped with a Balun. Balun is a transformer which converts a balanced line to unbalanced line and it is used in connecting a balanced antenna element with an unbalanced co-axial line [11]. Sleeve and tapered Balun designs are commonly used in implantable [10]-[12] and wearable [13] antennas systems. For sleeve Balun, a short-circuited sleeve around the coaxial cable in the form of a third cylindrical conductor is typically used to encompass the coaxial feed line. On the other hand, in tapered Balun design, the outer conductor is gradually tapered to make a smooth transition to a strip that has the same width as that of the inner conductor at the distal end. At the desired frequency of operation, the tapered transition should take place over at least a few wavelengths in length [10]. In any of these designs Balun will add an additional 
structure that increases the size or volume of the antenna. It is

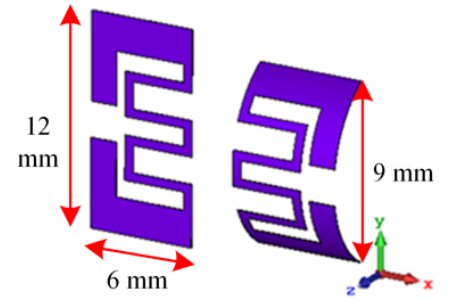

(a)

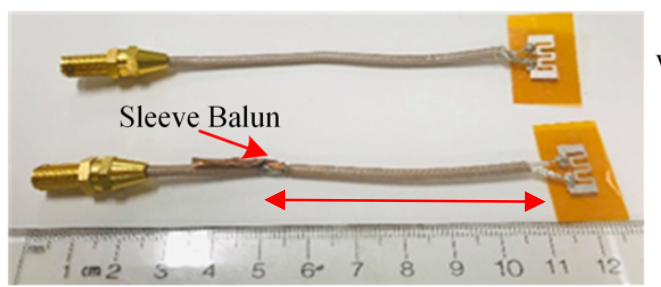

(c)

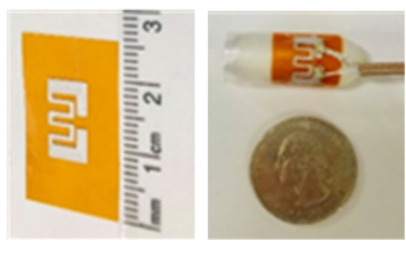

(b)

Antenna without Balun $\longleftarrow$

Antenna with Balun
FIGURE 1. Configuration of (a) the proposed flat and bent loop antenna, (b) fabricated flat and bent antennas, and (c) antennas with and without Balun.

important to consider the effect of Balun and how important it is to be integrated with the antenna. If there is a way of removal the Balun it will be desired.

The flexible antenna that is proposed in this paper features lightweight and compact size $\left(2.5 \mathrm{~mm}^{3}\right)$ that can be integrated with a radio frequency (RF) tag for tracking small animals such as small birds, mammals, or even insects. In addition, it can be implanted and used for animal's health monitoring applications. This antenna consists of an integrated sleeve Balun and a loop antenna. The antenna is similar to what we proposed in [14]. The remainder of the manuscript is organized as follows: Section II introduces the configuration of the proposed antenna integrated with a sleeve Balun along with materials used in the fabrication and measurement processes. Simulated and experimental results of the antenna in free-space and phantom environments are presented in Section II. Conclusion remarks are given in Section IV. All the simulations were conducted using CST Microwave Studio (MWS) [15].

\section{ANTENNA DESIGN AND MATERIALS}

The proposed antenna is a meandered loop with surface dimensions of $12 \times 6 \mathrm{~mm}^{2}$ printed on a $170 \mu \mathrm{m}$ thick Kapton substrate with permittivity $\left(\varepsilon_{\mathrm{r}}\right)$ and loss tangent $(\tan \delta)$ of 3.5 and 0.0027 at $1 \mathrm{GHz}$, respectively. The material is flexible, low profile, and biocompatible, which satisfies the requirements of wearable and implantable antennas. The antenna was printed on this substrate using Voltera-V One printer [16]. The antenna was folded and adhered to the outer surface of an air-filled cylindrical model of $11 \mathrm{~mm}$ diameter and $26 \mathrm{~mm}$ length to achieve the conformal antenna. The antenna is fed by a coaxial line and a sleeve Balun is designed at the center frequency. The Balun structure is realized by encompassing the feeding cable with a hollow circular conductor of diameter $3 \mathrm{~mm}$, and length of $22 \mathrm{~mm}$. The integrated conductor and the outer

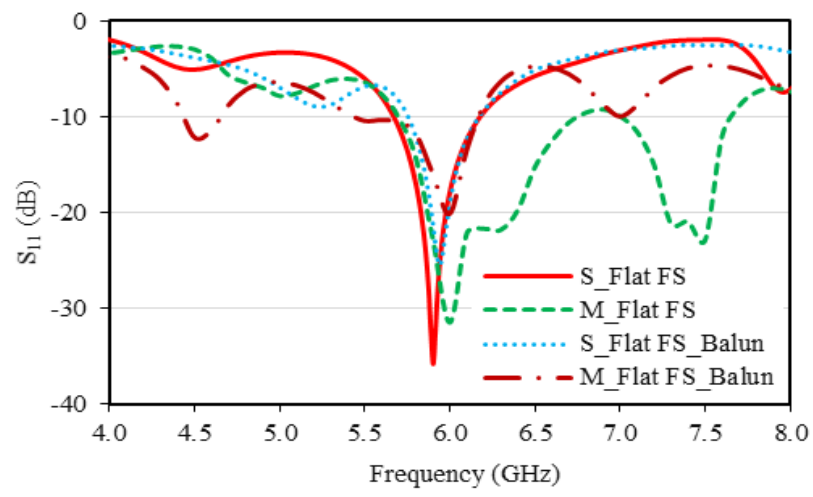

(a)

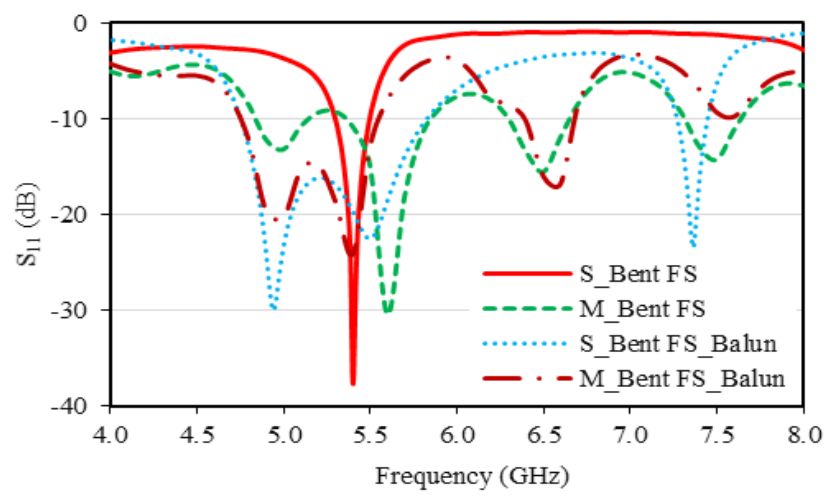

(b)

FIGURE 2. Reflection coefficients $\left(\mathrm{S}_{11}\right)$ of (a) flat and (b) bent flexible antenna in free-space (FS) based on simulation (S) and measurement (M) results.

conductor section of the enclosed coax feed constitute a new coaxial line for the outer-surface currents of the feed line. The length and termination at the proximal end of the new transmission line can be properly chosen to create a choke point with high impedance at its distal end to effectively suppress the outer surface currents. The sleeve Balun is placed at $\sim 5 \mathrm{~cm}$ away from the antenna, which is equivalent to $1 \lambda$ at the resonance frequency. Prototypes of fabricated antennas are shown in Fig. 1. For the antenna's detailed dimensions, please refer to [14]. For testing the implanted antenna a muscle-mimicking phantom was made in a mold with the same dimensions of $50 \times 50 \times 50 \mathrm{~mm}^{3}$ as those used in the simulations. Details of phantom preparation can be found [17].

\section{SIMULATED AND EXPERIMENTAL RESULTS}

\section{A. WEARABLE ANTENNA: FREE-SPACE ENVIRONMENT}

Reflection coefficients $\left(\mathrm{S}_{11}\right)$ of the flat and bent antenna in a free-space environment are shown in Fig. 2. For the flat antenna, input impedance bandwidth (i.e., $\mathrm{S}_{11}<-10 \mathrm{~dB}$ ) of $540 \mathrm{MHz}(5.65-6.19) \mathrm{GHz}$ centered at $5.9 \mathrm{GHz}$ is achieved based on simulation results. The antenna's 
bandwidth covers the Industrial, Scientific, and Medical (ISM) $5.8 \mathrm{GHz}$ band for wearable devices. Keysight

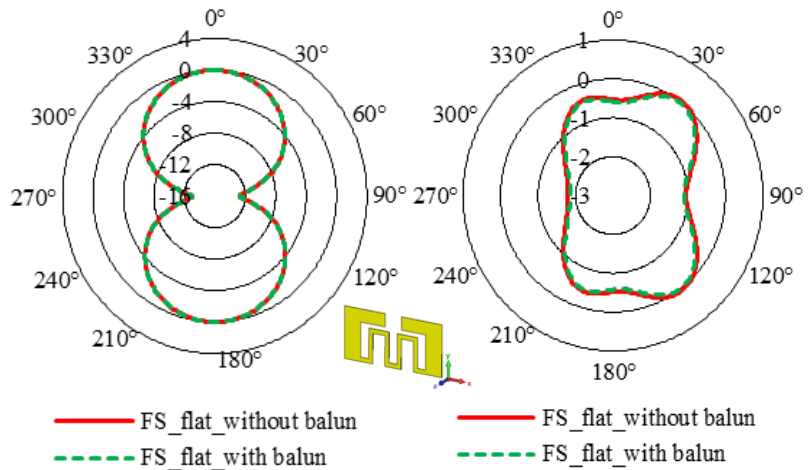

(a)

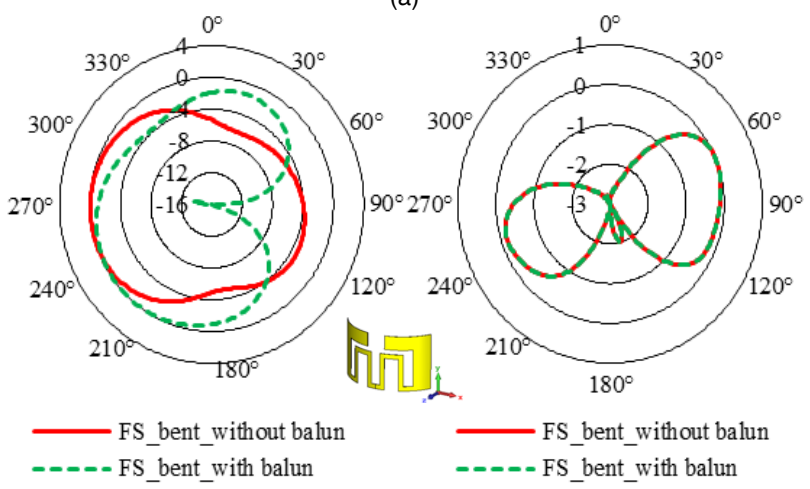

(b)

FIGURE 3. Simulated radiation patterns of (a) flat and (b) bent flexible antenna with and without a Balun in free-space (FS) at resonance frequencies in $\phi=0^{\circ} \mathrm{H}$-plane (left) and $\phi=90^{\circ} \mathrm{E}$-plane (right).

E5071C vector network analyzer $(300 \mathrm{kHz}-20 \mathrm{GHz}$ frequency operating range) was used for the S-parameter measurements. A wider input impedance bandwidth of 1.1 $\mathrm{GHz}$ and a slight shift in the resonance frequency from 5.9 $\mathrm{GHz}$ to $6 \mathrm{GHz}$ are observed based on measurement results. However, we observed that integration of a sleeve Balun at the center frequency in the antenna design resulted in a matching bandwidth with the simulation results with a reduction in the $S_{11}$ level. It is worth mentioning that tolerances introduced in the fabrication process cause discrepancies between simulation and measurement results.

In a practical wearable application, the flexible antenna may change its shape and dimensions due to conformability with the surfaces. Therefore, structural deformation conditions such as bending are considered. In such conditions, resonance frequency and $S_{11}$ need to be evaluated since they are prone to change due to impedance mismatch and change in the effective electrical length of the radiating element [18]. As can be seen in Fig. 2(b), antenna bending resulted in a shift in the resonance frequency from $5.9 \mathrm{GHz}$ (flat) to $5.46 \mathrm{GHz}$ (bent) with a reduction in the antenna bandwidth, based on simulation results. On the other hand, based on measurements, the antenna exhibits a wider impedance bandwidth of $1 \mathrm{GHz}$ $(4.90-5.90) \mathrm{GHz}$ with multiple resonances at $5 \mathrm{GHz}$ and
5.6 GHz. Despite our best effort, differences in the antenna bending in measurements and simulations are unavoidable,

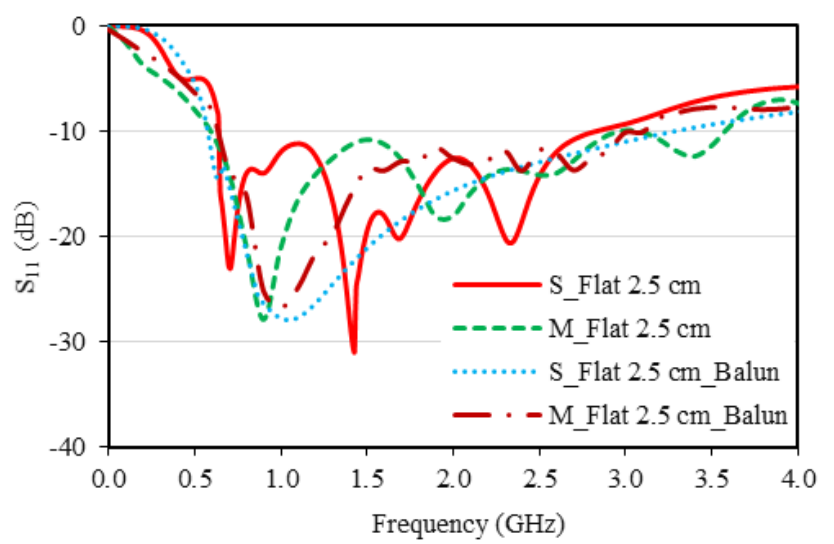

(a)

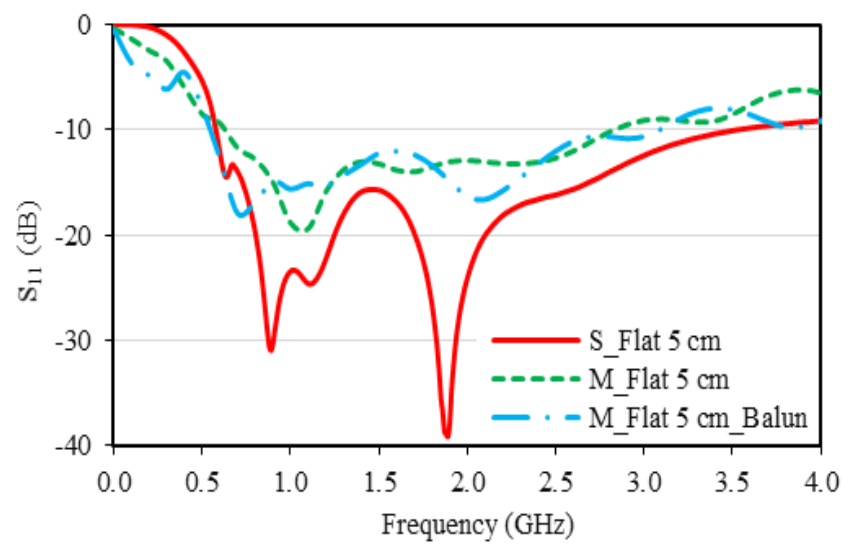

(b)

FIGURE 4. $S_{11}$ of flat flexible antenna implanted in muscle phantom at (a) $2.5 \mathrm{~cm}$ and (b) $5 \mathrm{~cm}$ depths based on simulation (S) and measurement (M) results.

since it is impossible to reach an ideal uniformity of the bending radius across the antenna structure as accurately as in simulations [19]. However, we noticed that Balun integration in the bent antenna resulted in a similar input matching behavior of a wider bandwidth and multiple resonances as of antenna without a Balun.

Radiation patterns on the antenna's E-plane and H-plane at resonance frequencies are shown in Fig. 3 for the antenna in free-space. Balun integration with the antenna in flat form did not affect the radiation patterns. On the other hand, when the antenna is bent, integration of the balun shows changes in the H-plane radiation pattern by adding a null point along $\varphi=90^{\circ}$. However, the beamwidth and location of the maximum gain point are not affected.

\section{B. IMPLANTABLE ANTENNA: PHANTOM ENVIRONMENT}

To address the effects of Balun integration in the antenna design in the case of an implanted antenna in a high loss material surrounding, the antenna is evaluated when inserted inside a muscle mimicking phantom. The electrical 
properties of the tissue phantom were measured using an 85070E Keysight high-performance dielectric probe.

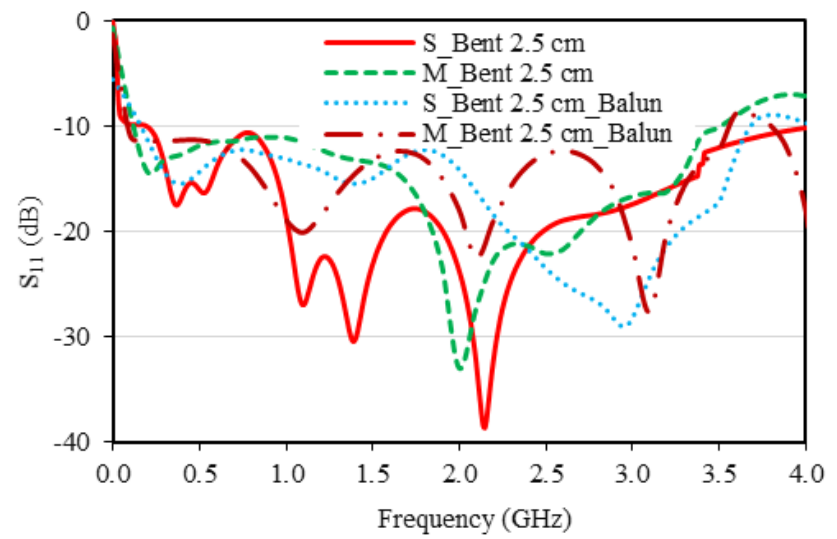

(a)

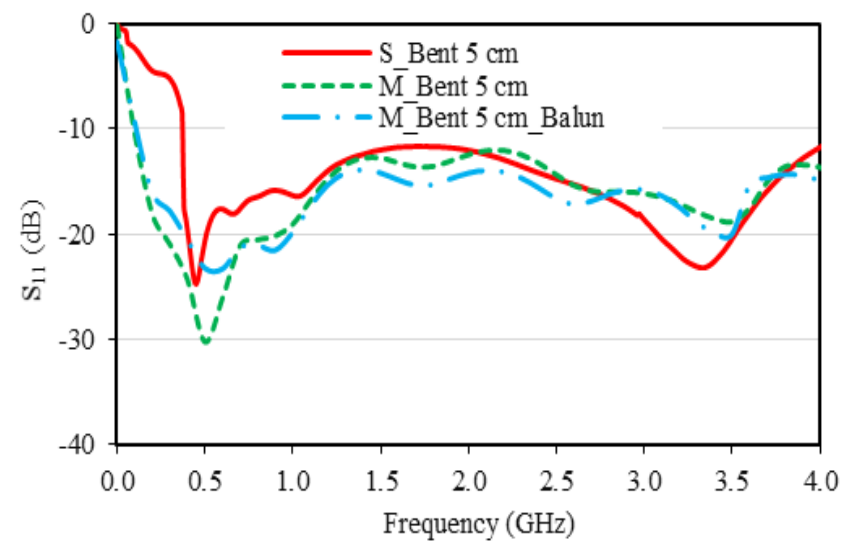

(b)

FIGURE 5. $S_{11}$ of bent flexible antenna implanted in muscle phantom at (a) $2.5 \mathrm{~cm}$ and (b) $5 \mathrm{~cm}$ depths based on simulation (S) and measurement (M) results.

$\mathrm{S}_{11}$ results of the implanted antenna are shown in Figs. 4 and 5 , for two implantation depths $(2.5 \mathrm{~cm}$ and $5 \mathrm{~cm})$, when the antenna was in flat and bent forms, respectively. Implanting the antennas in the animal's body for health monitoring and tracking purposes is advantageous because the measured signals will not be affected by the environment and the sensors will not get lost due to animals' movement.

In general, the implanted antenna has a wide bandwidth characteristic. Another advantage seen here is the negligible effect of Balun on the implanted antenna's matching, which means by removing the Balun the system can have a smaller volume. In this case, the losses of the tissue act as a Balun and suppresses the currents on the outer side of the cable. Furthermore, radiation patterns on the antenna's E-plane and $\mathrm{H}$-plane at resonance frequencies are studied and shown in Fig. 6, for an antenna implanted at $2.5 \mathrm{~cm}$ depth. Balun integration with the implanted antenna did not cause significant changes in the radiation patterns. It is worth mentioning that the radiation properties of the implanted antenna without Balun at $2.5 \mathrm{~cm}$ and $5 \mathrm{~cm}$ implantation depths are studied comprehensively in [14].

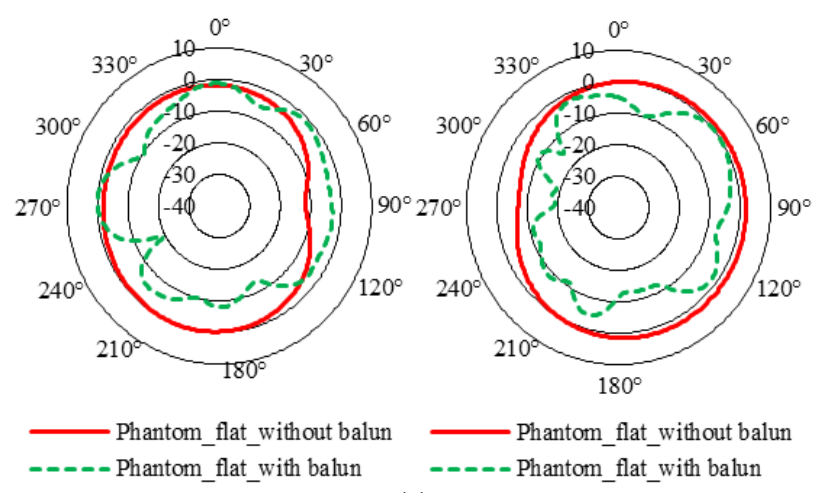

(a)
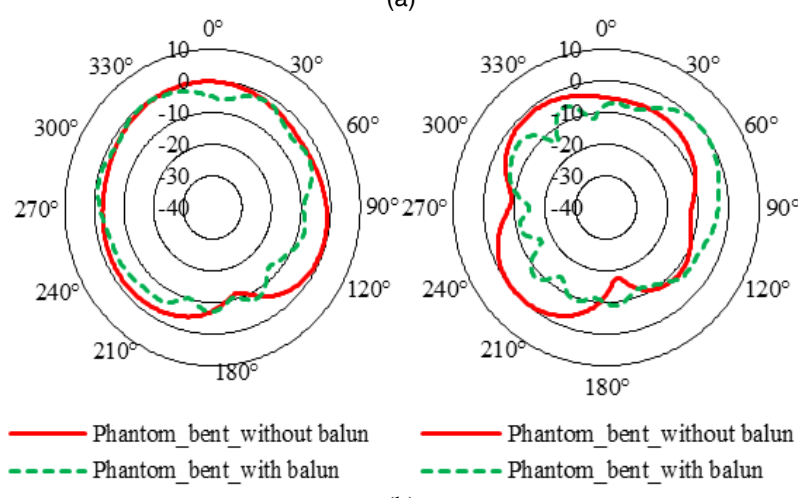

(b)

FIGURE 6. Simulated radiation patterns of (a) flat and (b) bent flexible antenna with and without Balun in phantom (2.5 implantation depth) at resonance frequencies in $\phi=0^{\circ} \mathrm{H}$-plane (left) and $\phi=90^{\circ} \mathrm{E}$-plane (right).

\section{v. CONCLUSION}

In this paper, a small size flexible antenna was considered and the need for Balun for its application in animal tracking and health-monitoring systems was studied. A prototype of the proposed design was fabricated and experimentally characterized in free-space and muscle mimicking phantom. Experimental results indicate that in a free-space environment, Balun integration with a flat antenna resulted in expected bandwidth and operating resonance frequency as in simulations. When the antenna was bent the addition of Balun enhanced antenna matching properties providing a wider bandwidth of $1 \mathrm{GHz}(4.90-5.90) \mathrm{GHz}$ compared to simulated bandwidth of $240 \mathrm{MHz}(5.35-5.56) \mathrm{GHz}$ and multiple resonances behavior at $5 \mathrm{GHz}$ and $5.6 \mathrm{GHz}$. For implanted antenna in planar and conformal arrangements at two implantation depths $(2.5 \mathrm{~cm}$ and $5 \mathrm{~cm})$, high loss tissue acted as a Balun and suppressed the current on the outer side of the cable therefore the Balun did not show much effect. Hence, our conclusion is to reduce the size of the implanted antenna, the Balun can be removed. 


\section{ACKNOWLEDGMENT}

The authors would like to acknowledge the assistance of Jon Debeltz and Mohammad Haerinia in different steps of antenna fabrication and testing. Tafila Technical University and the University of North Dakota are acknowledged for their financial support.

\section{REFERENCES}

[1] J. N. Wu, Z. Q. Zhao, J. Z. Liu, Z. P. Nie, and Q. H. Liu, "A compact linear tapered slot antenna with integrated Balun for UWB applications," Prog. Electromagn. Res. C, 2012, doi: 10.2528/PIERC12031204.

[2] R. Brugarolas et al., "Wearable heart rate sensor systems for wireless canine health monitoring," IEEE Sens. J., 2016, doi: 10.1109/JSEN.2015.2485210.

[3] N. A. Mungroo and S. Neethirajan, "Biosensors for the detection of antibiotics in poultry industry-A Review," Biosensors. 2014, doi: 10.3390/bios4040472.

[4] K. Telve, M. Mägi, J. Lodjak, P. Kilgas, J. Remm, and R. Mänd, "Looking at the forest through the eyes of birds: A radio-tracking study of microhabitat use in provisioning great tits," Acta Oecologica, 2020, doi: 10.1016/j.actao.2020.103531.

[5] F. Dressler et al., "From radio telemetry to ultra-low-power sensor networks: Tracking bats in the wild," IEEE Commun. Mag., 2016, doi: 10.1109/MCOM.2016.7378438.

[6] A. H. H. Nograles and F. S. Caluyo, "Wireless system for pregnancy detection in cows by monitoring temperature changes in body," in Proceedings - 2013 IEEE 9th International Colloquium on Signal Processing and its Applications, CSPA 2013, 2013, doi: 10.1109/CSPA.2013.6530005.

[7] A. Jukan, X. Masip-Bruin, and N. Amla, "Smart computing and sensing technologies for animal welfare: A systematic review," $A C M$ Computing Surveys. 2017, doi: 10.1145/3041960.

[8] S. Neethirajan, "Recent advances in wearable sensors for animal health management," Sensing and Bio-Sensing Research. 2017, doi: 10.1016/j.sbsr.2016.11.004.
[9] H. Tang, J. X. Chen, H. Chu, G. Q. Zhang, Y. J. Yang, and Z. H. Bao, "Integration design of filtering antenna with load-insensitive multilayer Balun filter," IEEE Trans. Components, Packag. Manuf. Technol., 2016, doi: 10.1109/TCPMT.2016.2600541.

[10] H. Luyen, S. C. Hagness, and N. Behdad, "A minimally invasive coax-fed microwave ablation antenna with a tapered Balun," IEEE Trans. Antennas Propag., 2017, doi: 10.1109/TAP.2017.2755258.

[11] S. Chatterjee, S. Suresh Kumar, A. N. Raut, K. Sakthidasan Sankaran, and P. M. Rubesh Anand, "A wide band Balun design using metamaterial technique for low frequency antenna application," in Proceedings of the 2019 IEEE International Conference on Communication and Signal Processing, ICCSP 2019, 2019, doi: 10.1109/ICCSP.2019.8697948.

[12] X. T. Yang, H. Wong, and J. Xiang, "Polarization reconfigurable planar inverted-F antenna for implantable telemetry applications," IEEE Access, 2019, doi: 10.1109/ACCESS.2019.2941388.

[13] M. R. Islam and M. Ali, "A $900 \mathrm{MHz}$ beam steering parasitic antenna array for wearable wireless applications," IEEE Trans. Antennas Propag., 2013, doi: 10.1109/TAP.2013.2269764.

[14] A. Alemaryeen, "Design of a miniaturized wideband antenna for wireless capsule endoscopy system," Appl. Phys. A Mater. Sci. Process.

[15] CST Microwave Studio [Online]. Available: http/www.cst.com. [Accessed: January, 2019].

[16] Volter https://www.voltera.io/product/technology/. [Accessed: February, 2019].

[17] D. F. Sievenpiper et al., "Experimental validation of performance limits and design guidelines for small antennas," IEEE Trans. Antennas Propag., 2012, doi: 10.1109/TAP.2011.2167938.

[18] A. Alemaryeen and S. Noghanian, "On-body low-profile textile antenna with artificial magnetic conductor," IEEE Trans. Antennas Propag., 2019, doi: 10.1109/TAP.2019.2902632.

[19] S. Noghanian and M. Griesi, "On the accuracy of flexible antennas simulations," Appl. Comput. Electromagn. Soc. J., 2020, doi: 10.13140/RG.2.2.25003.05920. 